HUTP-95/A047, IASSNS-HEP-95/105

hep-th/9511222

\title{
D-Branes and Topological Field Theories
}

\author{
M. Bershadsky and C. Vafa \\ Lyman Laboratory of Physics, Harvard University \\ Cambridge, MA 02138, USA \\ and \\ V. Sadov \\ School of Natural Sciences and School of Mathematics, \\ Institute for Advanced Study \\ Olden Lane, Princeton, NJ 08540, USA
}

\begin{abstract}
In the presence of a D-brane a string theory develops a new subsector. We show that for curved D-branes the corresponding sector is a (partially twisted) topological field theory. We use this result to compute the degeneracy of 2-branes wrapped around $K 3$ cycles as well as 3-branes wrapped around CY threefold vanishing 3-cycles. In both cases we find the degeneracy is in accord with expectation. The counting of BPS states of a gas of 0 -branes in the presence of a 4 -brane in $K 3$ is considered and it is noted that the effective 0 -brane charge is shifted by 1 , due to a quantum correction. This is in accord with string duality and the fact that left-moving ground state energy of heterotic string starts at -1 . We also show that all the three different topological twistings of four dimensional $N=4$ Yang-Mills theory do arise from curved three-branes embedded in different spaces (Calabi-Yau manifolds and manifolds with exceptional holonomy groups).
\end{abstract}

Nov. 1995 


\section{Introduction}

A significant progress has been made in understanding type II string solitons by identifying the sources of Ramond-Ramond charges as D-branes [1]. As specific applications this has led to verification [2] of the prediction of $(n, m)$ strings as bound states as required by string duality [3] and connecting type IIB $S L(2, \mathbf{Z})$ duality in ten dimensions to the appearance of massless gauge particles and matter in type II compactifications on singular $K 3$ and Calabi-Yau threefolds [4] [5]. In addition a systematic count of some expected $D$-brane bound states on $T^{4}$ [6] [7] has become possible and has led to counting of some of the Hagedorn density of BPS states as ground state configurations for a supersymmetric gas of D-branes [8].

In most of the above applications, the world volume of the D-brane is flat. One aim of this paper is to study the setup for extending the above cases to more general cases where the world volume of the D-brane is curved. That this should be considered is natural when we recall that in the case of conifold on $K 3$ the enhanced gauge symmetry expected at those points [9] comes from a 2-brane wrapped around a vanishing $S^{2}$ and for the conifold point of a Calabi-Yau threefold, the massless charged hypermultiplet comes from a 3-brane wrapped around a vanishing $S^{3}$ [10].

Quite surprisingly we find that the new sector living on the worldbrane is automatically topologically twisted along the directions where the worldvolume is curved. This is a very natural and satisfying realization of topological field theories in the context of string theory. There is, however, a minor twist to the story: The bosonic fields which are no longer spin-0 due to twisting are partially compactified carrying the information about the embedding of the D-brane in the rest of the manifold.

The organization of this paper is as follows: In section 2 we discuss some generalities about the field theories on curved D-brane worldvolumes and their connection to topological field theories. In section 3 we consider the specific case of type II compactifications on $K 3$ and $T^{4}$. In particular we show that the degeneracy of the 2-branes on $K 3$ wrapped around a vanishing 2-cycle of $K 3$ is 1 , as expected. Moreover we show that there are no bound states corresponding to multiple wrappings. We also show that 2-branes wrapped around higher genus Riemann surfaces $\Sigma$ in $K 3$ are a natural candidate for the D-brane states corresponding to Hagedorn density of BPS states. We explicitly show this for genus one curves in $K 3$ (where genus $g$ corresponds to bosonic oscillator number $N_{L}=g$ ). In these cases we show that the relevant topological theory on $\Sigma \times \mathbf{R} \times S^{1}$ is of the form 
studied in [11] where we have a partially twisted (along $\Sigma$ ) $N=4$ theory. In section 4 we discuss the counting of bound states of $n$ 0-branes and one 4-brane on $K 3$ and show how it leads to the expected degeneracy on the heterotic side as suggested in [8]. A key feature of this correspondence is the appearance of an "anomaly" which causes a 4-brane to carry -1 unit of 0 -brane charge, thus shifting the effective value of 0 -branes $n \rightarrow n-1$.

In section 5 we discuss the counting for D-brane states for the Calabi-Yau conifold and we again find agreement with the prediction of the expected degeneracy [10]. In section 6 we give other examples including manifolds of exceptional holonomy $G_{2}$ and $\operatorname{Spin}(7)$ and Calabi-Yau fourfolds and discuss the implications of potential singularities for such cases. We find that all the three different twistings of $N=4$ topological theories are realized by this set of examples.

\section{D-branes and Topological Field Theories}

In this section we sketch the general connection between the D-branes and the topological field theories. In the presence of a D-brane, string theory develops a new subsector of open strings. The most intensively studied case corresponds to when the D-branes worldvolume is flat, in which case one finds that the corresponding field theory is the reduction of the $N=1$ Yang-Mills theory from 10 dimensions to $p+1$, where we are considering p-branes. However more general curved worldbranes can be considered. To see that this can be done, it suffices to recall how the D-branes were originally discovered by considering orientifolds [12] [13]: One considers modding out, in an oriented closed string theory, by a reflection in spacetime, which at the same time acts on the closed string by reversing its orientation. Suppose such a transformation has a fixed subspace in spacetime. Then we can consider open string states which end at these fixed subspaces as twisted sectors of this modding out. The fixed point subspace is to be identified with the worldbrane of a D-brane. The operation just sketched shows how one can obtain open strings by modding out closed string theories by identifying a $\mathbf{Z}_{2}$ transformation in the target space, with a worldsheet orientation reversal. Even if we are not discussing the unoriented string theories, and in particular wish to have orientable strings, this suffices to show that we can consistently couple D-branes to closed orientable strings. It also shows that the concept of D-branes is far more general than having a flat worldvolume. Consider an arbitrary string compactification and suppose it has a $\mathbf{Z}_{2}$ symmetry with a fixed curved subspace $F$. Then considering open strings whose boundary lies in $F$ gives a D-brane which preserves half the 
supersymmetries (left intact by the compactification). Note that for $\mathbf{Z}_{2}$ being orientation preserving in the target space, we would be talking about D-branes of type IIB and for orientation reversal, we would be talking about D-branes for type IIA strings. For example consider a Calabi-Yau hypersurface of dimension $n$, which is defined by an equation with real coefficients in $\mathbf{P}^{n+1}$. Then it admits a Ricci-flat metric which is invariant under the complex conjugation operation. Moreover the fixed point of the operation of complex conjugation can be identified with a real hypersurface in $\mathbf{R P}^{n+1}$ and thus defines a D-brane worldvolume consistent with preserving half the supersymmetries. A special case of the above that we will use in this paper is when a Calabi-Yau has a conifold type singularity which locally looks like

$$
z_{1}^{2}+\ldots+z_{n}^{2}=\mu
$$

Let us take $\mu$ to be real. Then the corresponding D-brane lies on the real sphere $S^{n}$.

From the above description one may think that we need Calabi-Yau manifolds with $\mathbf{Z}_{2}$ isometries to have non-trivial supersymmetric D-branes. This is not the case. The simplest way to see this is to start with a D-brane which comes from an orientifolding description and then deform the theory by marginal perturbations. These perturbations will interact in a supersymmetric way with the degrees of freedom on the D-brane and modify their shape precisely as to preserve half the supersymmetries. It is natural to speculate, given the simplicity of obtaining supersymmetric D-branes as deformations of orientifold planes, whether all of them can be viewed as obtained from such deformations.

Given the fact that the D-branes must preserve half the supersymmetries, and given the fact that their curved worldvolume does not generally support a covariantly constant spinor (as the above conifold example shows) we learn that their supersymmetry cannot be realized in the standard form- rather they must necessarily involve a twisted definition of supercharges. This means that the worldbrane theory is at least a partially twisted topological field theory! The partial twisting should make supersymmetry possible on the curved worldbrane, but lead to an ordinary (perhaps reduced) supersymmetry on the flat parts of the worldbrane, similar to what was considered in [11]. This is quite surprising because we did not artificially 'twist' the definition of supercharge. Nevertheless the consistent interaction of the D-brane with the rest of the string modes automatically does that for us! Had we studied the D-brane theory before introduction of topological field theories, we would have been forced to discover it by studying the realization of supersymmetry in the new subsector of string theory in the presence of D-branes. Thus 
topological field theories find their most natural interpretation in the setup of D-branes in string theory. In fact this also suggests that as long as the non-compact directions of a D-brane worldvolume has dimension less than or equal to 2, topological twisting in the rest of the dimensions is responsible for making sense of an otherwise meaningless field theory.

To understand this point about twisting and the unconventional Lorentz assignments to fields let us concentrate on the transformation properties of the scalars. We will also introduce a notation which we will use in the rest of the paper. Consider a type II string "compactification" given by $M^{9}$. We are using the word "compactification" to include $\mathbf{R}^{k}$ as part of $M^{9}$ for some $k$. Let $W^{p} \subset M^{9}$ be a $p$-dimensional spatial part of a p-brane. Then the bosonic degrees of freedom, living on the $p+1$ worldbrane $W^{p} \times \mathbf{R}$, consists of a gauge field $A$ on it, plus $9-p$ scalars. As pointed out in [2] changing the expectation values of scalars can be viewed as moving the D-brane in the ambient space. Clearly there are $9-p$ such directions which can be viewed as the 'light cone' degrees of freedom of the D-brane. However from this geometric description it is clear that they cannot be viewed as functions on $W^{p}$. Let us decompose the tangent bundle of $M$ on the subspace $W^{p}$ as

$$
T M=T W \oplus N_{W}
$$

where $N_{W}$ is the normal bundle on $W$. Note that the dimension of $N_{W}$ is $9-p$ and it is the correct number of degrees of freedom to describe the variation of $W$ within $M$. Thus the $9-p$ "scalars" are actually sections of this bundle. Twisting is forced on us by the geometry of the embedding. These scalars, in addition to transforming non-trivially under the holonomy group in $W^{p} \times \mathbf{R}$, may be partly compact. This is clear when we recall that they describe the movement of the D-brane in $M^{9}$ and $M^{9}$ may be partially compact. This will prove to be important for us to take into account properly.

We will be interested in counting degeneracy of D-brane states. Suppose we have a type II string compactified on

$$
M^{9}=\mathbf{R}^{9-d} \times X^{d}
$$

where $X$ is a compact $d$-dimensional manifold. To get a particle in the effective $\mathbf{R}^{10-d}$ spacetime, we have to wrap all the spatial components of the p-brane around a compact $p$-cycle $W^{p} \subset X$. We will consider the general situation of $N$ wrapped p-branes and look for possible bound states. The basic idea is the observation in [2] that, modulo the complications of bound states at threshold, the existence of bound states of D-branes 
is equivalent to having a mass gap in the corresponding $S U(N)$ QFT on the D-brane worldvolume (the $U(1) \subset U(N)$ part of it describes the center of mass degree of freedom). Moreover, if there is a gap, the degeneracy of the bound states is the same as the number of ground states in the corresponding QFT. However if we are looking for bound states of $N$ such p-branes at best we could have a bound state of the p-branes at threshold, because energetically the BPS bound state will lead to the same energy as $N$ static and separated wrapped p-branes.

To avoid the delicate issue of bound states at threshold it was suggested in [6] that if we indeed do have a bound state at threshold of $N$ p-branes, by considering a further compactification on a circle $S^{1}$, and considering a state of the BPS state with momentum $M$ around $S^{1}$, and if $M$ and $N$ are relatively prime, there would be no BPS subsystems with the same energy. In fact it is convenient to do a $T$-duality on the circle $S^{1}$ which maps the momentum mode to a winding mode and at the same time both exchange type IIA and type IIB strings and change p-branes to $(\mathrm{p}+1)$-branes incorporating the circle as a part of the worldvolume of the D-brane. The existence of a winding mode of $M$ units means, through the coupling of worldsheet $B$-field to the D-brane $U(N)$ gauge field [2] that we are turning on $U(1)$ electrical flux along $S^{1}$ which translates to a $\mathbf{Z}_{M}$ 't Hooft electrical flux in the $S U(N) \subset U(N)$. It is convenient to take the circle to be infinitely long, in which case we should have a mass gap in the corresponding field theory on the D-brane worldvolume $\mathbf{R}^{2} \times \Sigma^{p}$. From now on we will take for granted that we may talk about type II A(B) $p$-branes for $p$ even (odd) wrapped around $X$ or by the above transformation consider type IIB(A) compactification on $X \times S^{1}$ where now we have a $(\mathrm{p}+1)$-brane wrapped around $X \times S^{1}$. The reader should be aware of this transformation throughout this paper as we will be using either formulation depending on what is convenient for the question at hand. Note also we do not need to specify whether we are dealing with type IIA or B, because that is already implied by the parity of $\mathrm{p}$ for the p-branes under consideration.

We will also briefly consider the notion of D-instantons, where time is not part of the worldvolume of the D-brane. In other words, we also consider the case where the $p+1$ worldbrane is purely Euclidean. In such cases we would be mainly interested in compact worldbranes. These would be configurations relevant for non-perturbative corrections to string theory [14]. 


\subsection{Supersymmetric Cycles}

In this section we will discuss the conditions for having supersymmetric cycles. A supersymmetric cycle is defined by the condition that a worldvolume theory is supersymmetric [15] [16]. The $(p+1)$-cycle is supersymmetric if the global supersymmetry transformation can be undone by $\kappa$-transformation 1 , which implies that

$$
P_{-} \Psi=\frac{1}{2}\left(1-\frac{i}{(p+1) !} h^{-1 / 2} \epsilon^{\alpha_{1} \ldots \alpha_{p+1}} \partial_{\alpha_{1}} X^{m_{1}} \ldots \partial_{\alpha_{p+1}} X^{m_{p+1}} \Gamma_{m_{1} \ldots m_{p+1}}\right) \Psi=0,
$$

where $\Psi$ is a covariantly constant ten dimensional spinor, $h_{\alpha \beta}$ induced metric on p-brane and $\Gamma_{m_{1} \ldots . m_{p+1}}$ are ten-dimensional $\Gamma$-matrices. Supersymmetric 2- and 3-cycles on CalabiYau threefolds were considered in [16]. Supersymmetric 2-cycles are given by holomorphic curves. The condition on 3-cycles is more complicated. It requires that the pullback of the Käher form $\omega$ vanishes and the 3-volume is proportional to the pullback of the holomorphic 3-form $\Omega$, namely

$$
* \Phi(\omega)=0, \quad * \Phi(\Omega) \sim 1
$$

where $\Phi($.$) denotes the pullback and *$ is a Hodge dual on membrane world-volume. The cycles satisfying (2.3) minimize the p-brane volume [16].

It is a simple exercise 2 to find solutions of (2.3) for supersymmetric 2-cycles on K3. One finds that for a given Ricci-flat metric on $K 3$ the supersymmetric 2-cycles are holomorphic curves with respect to one of the complex structures compatible with metric. A family of such complex structures is parametrized by points on $\mathbf{P}^{1}$. It is easy to prove that for each element $e \in H_{*}(K 3, \mathbf{Z})$ with self-intersection $e^{2} \geq-2$ there is a choice of complex structure on $K 3$ for which it can be realized by a holomorphic curve of genus $e^{2} / 2+1$. From now on when we talk of supersymmetric 2-cycles on $K 3$ as holomorphic curves we implicitly assume the appropriate complex structure is chosen.

1 This should be the origin of topological twisting discussed in the previous section.

2 The moduli space of hyper-Kähler structures coincides with the space of 3-planes in $\mathbf{R}^{3,19}$. This 3-plane is spanned by three hyper-Kähler forms $\left\{J^{i}\right\}$ satisfying the quaternionic algebra. The condition (2.3) can be rewritten as

$$
\left(1-* \Phi\left(\sigma_{i} J^{i}\right)\right) \Psi=0
$$

where $\left\{\sigma_{i}\right\}$ are the three Pauli matrices. 
We will also be interested in supersymmetric cycles for compactification on $G_{2}$ and $\operatorname{Spin}(7)$ manifolds. These manifolds have been recently studied by Joyce [17] who constructed the first compact manifolds of these types. They have also been studied in conformal theory [18]. A $G_{2}$ holonomy manifold has a canonical 3-form $\phi$. There are two natural classes of cycles to consider, which have already been considered in the mathematics literature [19]: 3-cycles on which $\phi$ is the volume form (known as associative manifolds) and four dimensional submanifolds for which $* \phi$ is the volume form (coassociative manifolds). For $\operatorname{Spin}(7)$ manifold there is a canonical 4-form $\Phi$. A natural class of 4-cycles correspond to "Cayley" submanifolds, where $\Phi$ is the volume form of the 4-manifold. In each of these cases they minimize the volume in the corresponding class of the cycle and it is easy to see that they satisfy (2.3) and thus correspond to supersymmetric cycles. Examples of all such cycles were constructed in [17] using fixed points of $\mathbf{Z}_{2}$ isometries (just as our discussion above would have led us to search for).

\section{Type II Strings on $K 3$ and $T^{4}$}

In this section we shall discuss aspects of 2-brane states for compactification of type II strings on $K 3$ and on $T^{4}$. In the next section we shall connect this to bound states of 0-branes and a 4-brane.

\subsection{2-branes on $K 3$}

We consider 2-branes whose spatial degrees of freedom correspond to $W^{2}=\Sigma$ where $\Sigma$ is a Riemann surface of genus $g$ embedded "supersymmetrically" in $K 3$, which as discussed before means holomorphically embedded. Suppose we have $N$ coincident 2-branes. As usual we do a duality transformation by introducing an extra circle, changing 2-branes to 3-branes and ask what is the corresponding field theory on the 4-dimensional worldvolume of the 3-brane. Were it not for the curvature in $\Sigma$ the field theory living on the 3-brane worldvolume would have been the reduction of $N=1$ Yang-Mills theory with $U(N)$ gauge symmetry from 10 dimensions down to 4, i.e. an N=4 Yang-Mills theory in four dimensions. However as discussed in the previous section we must have a partially twisted version of $N=4$ theory. The easiest way to find which version is to consider the transformation properties of the scalars. As discussed in section 2, they come from the normal bundle $N_{W}$ which in this case is 6 dimensional. The normal bundle has two pieces. One comes from fixing the 4 coordinates on $\mathbf{R}^{4}$ which gives 4 real scalars as the normal bundle (i.e. a 
position in $\mathbf{R}^{4}$ ). The other 2 real or 1 complex component comes from the normal bundle $N_{\Sigma}$ of $\Sigma$ in $K 3$. Let us decompose the complex rank 2 tangent bundle $\mathcal{T}$ of $K 3$ along $\Sigma$ as $K_{\Sigma}^{-1} \oplus N_{\Sigma}$ where $K_{\Sigma}^{-1}$ is the tangent bundle to $\Sigma$. Then take the determinant, $\left.\operatorname{det} \mathcal{T}\right|_{\Sigma}=K_{\Sigma}^{-1} N_{\Sigma}$. Since the canonical bundle $\operatorname{det} \mathcal{T}$ of $K 3$ is trivial, we learn that the normal bundle is the cotangent bundle on $\Sigma: N_{\Sigma}=K_{\Sigma}$ and so we can think of the corresponding complex scalar to be a 1 -form on $\Sigma$. This is precisely the same partial topological twist of $N=4$ along $\Sigma$ considered in [11]. Let us denote the scalar fields which are 1-forms on $\Sigma$ by $\Phi_{z}$ and $\overline{\Phi_{\bar{z}}}$. Then the result of [11] implies that to find the light degrees of freedom of the gauge theory on $\mathbf{R}^{1} \times S^{1}$, we first have to solve the Hitchin equation

$$
\begin{gathered}
F_{z \bar{z}}=\left[\Phi_{z}, \overline{\Phi_{\bar{z}}}\right] \\
\bar{D}_{\bar{z}} \Phi_{z}=D_{z} \overline{\Phi_{\bar{z}}}
\end{gathered}
$$

where for $N$ three-branes, the gauge fields and the scalars are in the adjoint of $U(N)$. Let $\mathcal{M}^{H}$ be the moduli of solutions to these equations. Then as a piece of the effective theory on $\mathbf{R} \times S^{1}$ we would get a supersymmetric sigma model on $\mathcal{M}^{H}$. If the gauge dynamics on the 2-dimensional subspace can be integrated out, as would be the case for turning on ' $t$ Hooft magnetic flux on $\Sigma$ where the gauge field is completely broken or if we have only one threebrane where the gauge group is $U(1)$, there would be no additional degrees of freedom. Otherwise we will have in addition a $U(N)$ gauge theory on $\mathbf{R} \times S^{1}$ with $N=4$ supersymmetry (i.e. the field content of the reduction of 4 dimensional $N=2$ supersymmetric YM down to 2 dimensions). A case of particular interest is when we have only one threebrane. Then we have only a $U(1)$ gauge group, and the above equations simplify: They correspond to choosing a flat $U(1)$ bundle on $\Sigma$ and choosing in addition a harmonic 1-form corresponding to solutions of the scalar $\Phi$. Recalling the meaning of $\Phi$ as normal variation of $\Sigma$ in $K 3$, it implies that a choice of the harmonic 1-form is the same as a choice of an infinitesimal deformation of $\Sigma$ to a nearby holomorphic curve in $K 3$. In fact this description "compactifies" the Hitchin equation. In other words the higher order terms in the effective theory of the worldbrane retain the information about the meaning of $\Phi$ as a normal variation of $\Sigma$ in $K 3$. Thus the actual moduli space we end up with will be a kind of "compactification" of $\mathcal{M}^{H}$. What this means concretely for us is that this space is the same as the moduli of holomorphic curves of genus $g$ with a choice of a flat $U(1)$ bundle, i.e. a point on the Jacobian of the Riemann surface. Let us denote this space for the case of genus $g$ curve by $\mathcal{M}_{g}^{H}$. 
This space can be studied mathematically, see for example [20]. By definition, $\mathcal{M}_{g}^{H}$ is fibered over the space of holomorphic genus $g$ curves on $K 3$. The space of such curves can be identified as $\mathbf{P} H^{0}(\mathcal{O}(D))=\mathbf{P}^{g}$, where $H^{0}(\mathcal{O}(D)$ consists of functions with a simple pole on the divisor $D$ represented by curve of genus $g$. The fiber of $\mathcal{M}_{g}^{H}$ over a point on $\mathbf{P}^{g}$ is a Jacobian of the corresponding curve. This space has complex dimension $2 g$, it is hyperkähler and has the structure of a completely integrable system.

Let us consider the two simplest examples. For $g=0$, the holomorphic (rational) curves on $K 3$ are isolated. Moreover the Jacobian for $g=0$ is trivial, so $\mathcal{M}_{0}^{H}$ is a point. Equivalently, there is no non-trivial solution to (3.1). This is also true even if we consider $U(N)$ instead of $U(1)$, a fact we shall use later.

For $g=1$, the space of elliptic curves in $K 3$ is $\mathbf{P}^{1}$. The Jacobian of the elliptic curve coincides with the curve itself. Generically the curve is smooth, but there are 24 degenerate fibers which are rational curves with a double point. Such fibration over $\mathbf{P}^{1}$ fits together to form a $K 3$ ! Thus $\mathcal{M}_{1}^{H}=K 3$.

For $g>1$, we will now prove $e^{3}$ that a space of curves on $K 3$ with line bundles of degree $g$ is birationaly equivalent to a symmetric product $\operatorname{Sym}^{g}(K 3)$. A point on $\mathcal{M}_{g}^{H}$ may be viewed as a curve in $K 3$ together with $g$ unordered points on $\Sigma$ (we use an isomorphism $\left.\mathrm{Jac}_{g}=\operatorname{Sym}^{g}(\Sigma)\right)$. Forgetting the curve gives $g$ points in $K 3$, i.e. a point of $\operatorname{Sym}^{g}(K 3)$. Now fix a point on $K 3$. All curves of genus $g$ passing through this point are parameterized by a hyperplane in $\mathbf{P}^{g}$. For $g$ points on $K 3$ one gets $g$ hyperplanes in $\mathbf{P}^{g}$, which always intersect, genericaly in a point. Thus for any $g$ points on $\mathrm{K} 3$ there is at least one (genericaly one) curve of genus $g$ passing through them. Tautologicaly this curve has $g$ marked points on it 1 . This proves the birational equivalence. It is easy to see that the map is symplectic.

This theorem is not quite what we need. It may be viewed as a mathematical motivation for a conjecture that $\mathcal{M}_{g}^{H}$ is actualy related to $\operatorname{Sym}^{g}(K 3)$ and has the same cohomology in particular. A proof of this conjecture would involve analysis of singularities of both varieties using a theorem due to Lazarsfeld [22].

3 We thank E. Witten for the illuminating remark which contained the idea of this proof.

4 This is very much like the $N=2$ strings on $K 3$ [21] where the $N=2$ string computes the 'number' of holomorphic maps from genus $g$ Riemann surface to $K 3$. In fact in the context of type IIB theories and Euclidean 1-branes, the analogy may become more exact. Note in particular that $N=2$ string also has a $U(1)$ gauge bundle on the worldsheet, as is required for the D-brane. This suggests that $N=2$ string (and perhaps other topological strings as well) may be viewed as the theory of appropriately embedded Dirichlet 1-branes. 
Note that the number of D-brane states is the same as the dimension of the ground state for the worldbrane theory, which for one threebrane case is the same as the number of cohomology elements of $\mathcal{M}_{g}^{H}$. Type II-heterotic duality in 6 dimensions predicts this number to be the same as the degeneracy of the bosonic oscillation states with $N_{L}-1=\frac{1}{2} P^{2}$ where $P^{2}$ denotes the self-intersection of the 2 -brane part of the 3 -brane in $K 3$, i.e. $P^{2}=\Sigma \cdot \Sigma=2 g-2$. In other words we expect a degeneracy of bosonic oscillators for level $N_{L}=g$. This is in accord with what we mentioned for $g=0$, where the sigma model is trivial, and $g=1$, where the sigma model is the one on $K 3$ and so has dimension 24. In addition the $U(1)$ center of mass system gives rise to the expected supersymmetry degeneracy. Moreover for the higher values of $g$ the mathematical conjecture about the form of $\mathcal{M}_{g}^{H}$ is in accord with the suggestion made in [8] as to how the degeneracy of Hagedorn density of BPS states may arise. In fact we will see in the next section, after uncovering an anomaly, how the counting proposed in [8] for $n$ 0-branes and one 4 -brane gives the answer predicted by string duality. Thus the $T$-duality of string theory, which maps the 0 -brane-4-brane configuration to 2-brane configuration, would show the sensibility of the above mathematical conjecture about the structure of $\mathcal{M}_{g}^{H}$.

The case when $\Sigma$ is a 2 -sphere is special. This is the case when the cycle can vanish by varying the moduli of $K 3$, and so could lead to massless particles. One of the predictions of string duality is that when this happens for a D-brane wrapped around the 2-sphere we have exactly one BPS state, which is what we have found above, namely $\mathcal{M}_{0}^{H}$ is a point. There is a further prediction: If we take the D-branes to wrap around the 2-sphere $N$ times, or in other words, if we have $N$ coincident 2-branes, they should not form a bound state, otherwise we would end up with more massless modes than is expected on the heterotic side. The mathematical counterpart of this statement is quite clear: A cycle which is $N>1$ times 2 -sphere has self-intersection number equal to $-2 N^{2}<-2$. Hence it cannot be realized by a smooth holomorphic curve, or a supersymmetric 2 -cycle. We will now confirm that this is indeed the case.

As mentioned above, when we have $N$ D-branes, we have a $U(N)$ gauge group to deal with. Since the Hitchin equation in the case of the $S^{2}$ has a trivial solution, the gauge symmetry is unbroken and so in the remaining 2 dimensional theory we obtain an $N=4 U(N)$ gauge theory (with no extra matter). Modding out the center of mass degree of freedom, we will concentrate on the $S U(N)$ gauge symmetry and as discussed in section 2 we will consider subsectors with $\mathbf{Z}_{M}$ electrical flux and look for mass gap. The absence of mass gap will show that we will not have a bound state (and in fact implies that 
supersymmetry is broken in this subsector). As noted in [2] the usual anomaly matching conditions of ' $\mathrm{t}$ Hooft imply that for $N=4 \mathrm{YM}$ in $d=2$, unlike the $N=8$ case, there is no mass gap (to see that note that the $R$ symmetry group is $O(4)$ and the left-moving fermions can be chosen to transform as $(2,1)$ and right-moving ones as $(1,2)$. Non-triviality of the anomaly matching in any of the $S U(2) \subset O(4)$ implies the lack of mass gap in this theory). This implies that, as expected from string duality, $N$ 2-branes wrapped around $S^{2}$ do not form a bound state.

\subsection{Bound states and $U(n)$ Hitchin systems.}

Here we would like to discuss the situation with the bound states corresponding to supersymmetric 2-cycles in $X$, where $X$ is either $K 3$ or $T^{4}$. It was already explained that the rational cycles, which have self-intersection -2 , do not form bound states. The situation is however different for the cycles of genus $g>1$. The point is that a class $e \in H_{*}(X, \mathbf{Z})$ which is a multiple of another class $e=n \cdot e^{\prime}, n \in \mathbf{Z}_{+}$can be represented either by a smooth curve of genus $g$ or an n-tuple of coinciding curves of genus $g^{\prime}, g=n^{2}\left(g^{\prime}-1\right)+1$. The second representation allows us to think of such D-branes as bound states of simpler incident D-branes. Since this describes the same physical object, we should recover the same structure of moduli space using either description. This gives yet another interesting test of D-brane picture: The moduli space of curves of genus $g$ with $U(1)$ bundles was described above - it is a $2 g$-dimensional hyperkähler variety. On the other hand, following Witten, the moduli of n-tuples of curves of genus $g^{\prime}$ can be described as follows. A point on the moduli is a pair - a complex rank $n$ vector bundle $E$ on $\Sigma$ together with $n^{2}$ "scalars" $\phi_{z}^{i j}$. These "scalars" are sections of End $\mathcal{E} \times N_{\Sigma}$. They correspond to a condensate of D-strings connecting pairs of 2-branes. Such moduli space is indeed a hyperkähler variety of requisite dimension $2 g$ ! Locally one can think of it as a set of solutions of $U(n)$ Hitchin's system on curve of genus $g^{\prime}$. A similar problem was studied in some detail in [20]. There it is shown that indeed there is an isomorphism between the moduli of curves of genus $g$ with $U(1)$ and the moduli of n-tuples of curves of genus $g^{\prime}$ - a certain compactification of $U(n)$ Hitchin system. 


\subsection{Type II on $T^{4}$}

The situation for type II compactification on $T^{4}$ (or $\left.T^{4} \times S^{1}\right)$ and the resulting 2(3)branes is very similar to our discussion for the $K 3$ case, so here we highlight the differences.

It is not difficult to see using standard techniques that the moduli space of holomorphic curves of genus $g>1$ in $T^{4}$ is a product $\mathbf{P}^{g-2} \times T^{4}$. (Obviously, the $T^{4}$ factor accounts for the action of torus on itself.) If we consider curves together with flat $U(1)$ bundles, we get a hyperkähler variety of dimension $2 g$ fibered over $\mathbf{P}^{g-2} \times T^{4}$ by Jacobians $J_{g}=T^{2 g}$. For $g=1$ the space of elliptic curves in $T^{4}$ is $T^{2}$ and the space of elliptic curves with Jacobians is $T^{4}$. From above it follows that the moduli space for $g=2$ is $T^{4} \times T^{4}$. Clearly, two factors of $T^{4}$ correspond to the action of $T^{4}$ on the curve itself and on its Jacobian. In general, for $g \geq 2$ the moduli space have a structure $T^{4} \times X$ where in turn $X$ is a fibered product of $T^{4}$ over a $(2 g-4)$-dimensional hyperkähler manifold $Y$. Finally, $Y$ has a structure of a family of complex $(g-2)$-dimensional tori over $\mathbf{P}^{g-2}$.

For $g=3, Y$ is a hyperkähler 2-fold. Therefore, assuming compactness, this is either a torus $T^{4}$ or a $K 3$. Since it is fibered over $\mathbf{P}^{1}$, it has to be $K 3$. Thus the moduli space for $g=3$ is a product of $T^{4}$ and a $T^{4}$ bundle over $K 3$. In the next section we shall compare this answer with what is expected from string duality.

\section{Gas of 0-branes on $K 3$}

It was shown in [8] that a gas of $n$ 0-branes on $T^{4}$ in the presence of one 4-brane gives rise to a sigma model on $\left(T^{4}\right)^{n} / S_{n}$ where $S_{n}$ is the permutation group on $n$ objects. Moreover, the ground states of this system is as many as expected by string duality, i.e. left-moving superstring degeneracy at level $n$. This is based on the observation in 23] about the relation of the cohomology of this space to string oscillator partition function.

Let us briefly review the basic conclusion of [8], before considering the more involved case of $K 3$. By T-duality for $T^{4}$, a supersymmetric 2-cycle of genus $g$, with $g-1 \geq 0$, can be mapped into a sum of a fundamental 4-brane $\left[T^{4}\right]$ and $(g-1)$ 0-branes represented by points on $T^{4}$. The configuration space of such a system, taking into account that one 0-brane and one 4-brane form precisely one bound state [7] is $\mathrm{Sym}^{g-1}\left(T^{4}\right)$ coming from the position of the 0-brane, and a $T^{4}$ form the 4-brane (due to the choice of a flat $U(1)$

5 The result is in agreement with the result of [7] where two tori intersect at one point and lead to a degenerate genus 2 curve. 
background for the 4-brane). So finally the moduli space of the D-brane configuration we obtain is a product $T^{4} \times \operatorname{Sym}^{g-1}\left(T^{4}\right)$ in agreement with the prediction of $U$-duality [8]. This should reproduce the answer for the moduli space of 2-branes we obtained above!

This seems to be very likely, in view of the following facts. Both spaces are hyperkähler, of the same dimension $2 g$. Both have the product structure $T^{4} \times X$ where in turn $X$ is a $T^{4}$ bundle over a $(2 g-4)$-dimensional hyperkähler manifold $Y$. (This was explained above for the moduli of 2-branes. This is so here because $T^{4}$ acts freely on the symmetric product $\mathrm{Sym}^{g-1}\left(T^{4}\right)$ and $Y$ is defined as a quotient of this action.) Moreover, we can study explicitly three examples.

For zero (one) 0-brane on $T^{4}$ the answer is $T^{4}\left(T^{4} \times T^{4}\right)$ which agrees with what we had for the curves of genus one (two) on $T^{4}$. Let us see how the answer for two 0-branes on $T^{4}$ agrees with that for genus three curves. In this case $\operatorname{Sym}^{2}\left(T^{4}\right)$ has a singular locus on the diagonal $T^{4}$, with $A_{1}$ singularity in the transversal direction. We can blow up along the diagonal which amounts to creation of a smooth divisor $\mathbf{P}^{1} \times T^{4}$. There is a natural free action of $T^{4}$ on $T^{4} \times T^{4}:(a, b) \rightarrow(a+\xi, b+\xi)$. Symmetric group $S_{2}=\mathbf{Z}_{2}$ acts by $(a, b) \rightarrow(b, a)$. Let us consider the quotient $\left(T^{4} \times T^{4}\right) / T^{4}$. Obviously, this quotient is the 4-torus $T^{4}$ again, so one can think of $T^{4} \times T^{4}$ as a (nontrivial) fibered product of $T^{4}$ over $T^{4}$. An advantage of this point of view is immediate: the diagonal $T^{4}$ acts only on the fiber and, more importantly, $\mathbf{Z}_{2}$ acts only on the base. Since $T^{4} / \mathbf{Z}_{2}=K 3$ we proved that $Y=K 3$ so that $\operatorname{Sym}^{2}\left(T^{4}\right)$ is a $T^{4}$ bundle over $K 3$. This is exactly the result we obtained above for the genus 3 supersymmetric 2-cycles! For higher genus, the arguments given in the context of 2-branes on $K 3$ is presumably generalizable to the case of $T^{4}$.

It was suggested in [8] that the same idea should work in the context of bound states of 0 -branes and one 4-brane on $K 3$, in a way consistent with type II-heterotic duality, when we take into account the observation of [23] that $n$ copies of $K 3$ has the same degeneracy as bosonic string partition function at level $n$. In fact the gauge system in this problem is identical to that encountered in [8] in connection with the $T^{4}$ compactification, except for lacking the adjoint matter which came from the reduction of the 4-brane on $K 3$. In the case of $T^{4}$ this system was decoupled and its fermionic degrees of freedom provided some of the degeneracies expected for the BPS state. In our case they are absent, again in accord with the fact that we have a different degeneracy of BPS states expected. Everything works as in the $T^{4}$ case, except that when we have $m$ 0-branes and one 4-brane, the corresponding sigma model we get is on $\operatorname{Sym}^{m}(K 3)$, which has the same ground state degeneracy as the bosonic string with $N_{L}=m$. However the string duality in this case 
predicts that $N_{L}-1=m$ since $\frac{1}{2} P^{2}=m \cdot 1=m$. Thus there seems to be a shift of one unit unaccounted for.

Here we will argue that in the presence of a $K 3$ a 5 -brane acquires in addition - 1 unit of the RR charge corresponding to the two form. This shifts the $\frac{1}{2} P^{2} \rightarrow(m-1) \cdot 1=m-1$, which thus leads to the observed degeneracy in agreement with $N_{L}=m$. We will argue for this by making use of type IIB $S L(2, \mathbf{Z})$ duality in 10 dimensions, though it should also be possible to check it directly in string perturbation theory. Consider a 5-brane. We will argue that in the $6 \mathrm{~d}$ worldvolume of the 5 -brane there is an effective interaction of the form

$$
\frac{1}{48} \int_{M^{6}} B^{R} \wedge p_{1}
$$

where $B^{R}$ is the RR 2-form gauge field and is normalized to have periodicity $2 \pi$ and $p_{1}$ is the first Pontryagin class of the manifold. We will not be careful with signs, but this result shows that if we compactify the 5 -brane on a $K 3$ where $p_{1}=-48$, in the resulting $2 \mathrm{~d}$ theory, we have an effective one unit of RR 2-form charge. In other words a 5-brane compactified on a $K 3$ acquires an additional charge as if there were an anti-1-brane on K3. We will show that the absolute value of the shift is one, but as in [24] we will have to confess to not checking the sign of the additional charge.

The most straight forward way to check (4.1) would be to compute a three point function of the RR 2-form field with emission of two gravitons on a disc where the boundary of the disc is mapped to the worldvolume of the 5-brane. We have not done this, but have found an indirect argument for its existence by making use of type IIB $S L(2, \mathbf{Z})$ duality in 10 dimensions. Consider type IIB strings in the presence of $n$ parallel fivebranes. Then according to the prediction of $S L(2, Z)$ duality, by strong/weak duality this is equivalent to $D$-string (the $(0,1)$ string in the notation of [3]) in the presence of symmetric fivebrane of charge $n$, which according to the result of [5] is equivalent by T-duality to type IIA on $A_{n-1}$ ALE space. Now we recall that if we compactify type IIA on $K 3$ we induce a one-loop correction of the form [24]

$$
\frac{1}{2} \int_{M^{6}} B^{N S} \wedge p_{1}
$$

where $B^{N S}$ denotes the 2-form gauge field from the NS-NS sector. It is not too difficult to generalize the result in [24] to the case of ALE space $A_{n-1}$ where one finds a quantum correction of the form

$$
\frac{n}{48} \int_{M^{6}} B^{N S} \wedge p_{1}
$$


One way to see this is to recall that $K 3$ can be viewed as 24 cosmic strings [25] and $n$ cosmic strings make up an $A_{n-1}$ singularity (see [5] for a discussion of this). To see what this induced correction means in the original type IIB string theory (i.e. in the $(1,0)$ string) we recall that under $S L(2, \mathbf{Z})$ duality we have an exchange $B^{N S} \leftrightarrow B^{R}$. Thus we end up with the expected result (1.1).

\section{Calabi-Yau Conifold}

In this section we will briefly mention the situation with counting of BPS states for 3-branes wrapped around a vanishing 3-sphere near a Calabi-Yau conifold. The arguments are very similar to the $S^{2}$ 2-branes in $K 3$ and so we will be rather brief here. Clearly also other types of 3-cycles can be studied with the same techniques as used for higher genus curves in $K 3$, but we will not consider them in this paper.

In the 3-brane worldvolume we have $S^{3} \times \mathbf{R}$. Let us first note that since near the conifold the Calabi-Yau is approximated by $T^{*} S^{3}$, the 6 'scalars' will transform as a 1-form on $S^{3}$ and three scalars. Thus if we consider a single 3-brane, there is a unique vacuum, since there are no non-trivial flat connections on $S^{3}$ and moreover the dimension of the space of harmonic 1-forms is 0 (which implies that the $S^{3}$ is rigid). This implies that we have a single BPS state corresponding to 3-brane wrapping around the 3-cycle.

Now we show that there are no bound states when we wrap the 3 -brane $N$ times around $S^{3}$. Again the internal theory will have a trivial moduli space and we are reduced (modulo the center of mass degree of freedom) to a reduction of $S U(N) N=1, d=4$ system down to $d=1$. Again by the trick of [6] to avoid the complications of bound states at threshold we transform the problem to the $S U(N)$ gauge system in $d=2$ which has $N=2$. Again this system has no mass gap (as noted in [2]) using 't Hooft's anomaly matching condition. Thus there are no multiple bound states of the threebrane wrapped around the $S^{3}$ of the conifold, in agreement with the proposal in [10].

\section{6. $G_{2}$ and $\operatorname{Spin}(7)$ Manifolds and $N=4$ Topological Twists}

In this paper we have seen that in the worldvolume of a D-brane we end up with a twisted version of an $N=4$ theory. In this section we show that we can obtain all the three twistings of $N=4$ by considering different 3-branes embedded in appropriate manifolds. We will also point out the existence of potentially new massless solitons when 
we compactify on manifolds of $G_{2}$ and $\operatorname{Spin}(7)$ holonomy when appropriate 3 or 4 cycles vanish.

Let us recall that $N=4$ theory in $d=4$ has three inequivalent twists [23] depending on how we embed the Lorentz group $S U(2) \times S U(2)$ in the global symmetry of $N=4$ which is $S U(4)$. We can classify the embedding by how the fundamental 4 of $S U(4)$ decomposes. There are three cases 6 which will lead to a topological symmetry: i) $(\mathbf{2}, \mathbf{1}) \oplus$ $(\mathbf{1}, \mathbf{2})$ ii $)(\mathbf{1}, \mathbf{2}) \oplus(\mathbf{1}, \mathbf{2})$ iii $)(\mathbf{1}, \mathbf{2}) \oplus(\mathbf{1}, \mathbf{1}) \oplus(\mathbf{1}, \mathbf{1})$. The cases i) and ii) lead to 2 unbroken topological supersymmetries, whereas the case iii) leads only to 1 unbroken topological supersymmetry. Let us consider the transformation of the 'scalars' in each case. The scalars transform as the 6 of $S U(4)$, i.e. the second rank antisymmetric representation. Thus under the twisted Lorentz group the scalars now transform as

$$
\begin{array}{rr}
\text { i) } & 2(\mathbf{1}, \mathbf{1}) \oplus(\mathbf{2}, \mathbf{2}) \\
\text { ii) } & 3(\mathbf{1}, \mathbf{1}) \oplus(\mathbf{1}, \mathbf{3}) \\
\text { iii }) & 2(\mathbf{1}, \mathbf{1}) \oplus 2(\mathbf{1}, \mathbf{2})
\end{array}
$$

Let us consider a Euclidean 3-brane, i.e., we look for 4-brane configurations in compact spaces. These would serve as instantons for string theory. Then the above transformation of the scalars, combined with our discussions in section 2 imply that in cases i) and iii) we should look for an 8-manifold compactification, whereas in case ii) we should look for a 7-manifold compactification. Moreover taking into account the number of unbroken topological supersymmetries in each of these cases, we see that the candidates are fixed to be i) a Calabi-Yau 4-fold compactification, ii) $G_{2}$ holonomy 7 -manifolds, iii) $\operatorname{Spin}(7)$ holonomy 8-manifolds.

Example for case i) is easy to come by — we have essentially mentioned it in section 2, where we discussed Calabi-Yau compactification with complex conjugation symmetry. The 4-brane in this case will be the real 4-manifold and the normal bundle will be its cotangent bundle, so that the non-trivial scalar will be identifiable with 1-forms on it, i.e. transform as $(2,2)$ as expected for case i).

It turns out for coassociative four submanifolds in $G_{2}$ the normal bundle is indeed $(1,3)$ [26] as expected from the discussion above. For the $\operatorname{Spin}(7)$ case the normal bundle is of the form $S_{+} \otimes V$ where $S_{+}$is a spin bundle of a given chirality and $V$ is a two

6 There is a misprint in case i) above considered in [23. 
dimensional bundle [27]. When $V$ is trivial, this becomes $S_{+} \oplus S_{+}$, which is exactly what we had expected for case iii).

Now we come to the question of D-branes as solitonst. We will be brief because some of the mathematical aspects of moduli space of compactifications on $G_{2}$ and $\operatorname{Spin}(7)$ manifolds have not been worked out. However it is clear that if we have a vanishing associative, or coassociative or Cayley submanifolds, we will have a massless state. What is not clear yet is whether these will appear at finite distance in moduli space, and to what extent the moduli space metric receives worldsheet and quantum corrections. But clearly once this is known, the methods discussed above will be easily applicable in counting their bound states.

We would like to thank Andrei Johansen, Dominic Joyce and Tony Pantev and Edward Witten for valuable discussions. The research of V. S. is supported in part by NSF grant DMS 9304580. The research of M. B. and C. V. is supported in part by NSF grant PHY92-18167. The research of M. B. was also supported by NSF 1994 NYI award and DOE 1994 OJI award.

7 In the case of $\operatorname{Spin}(7)$ and Calabi-Yau fourfold compactifications note that to cancel the anomaly computed in [24] we need to turn on some $D$-branes on the manifold to stabilize it. This should be interesting to study further. 


\section{References}

[1] J. Polchinski, Dirichlet-Branes and Ramond-Ramond Charges, NSF-ITP-95-122, hepth/9510017

[2] E. Witten, Bound States Of Strings And p-Branes, hep-th/9510135

[3] J. Schwarz, An SL(2,Z) Multiplet of Type IIB Superstrings, CALT-68-2013, hepth/9508143

[4] M. Bershadsky, V. Sadov and C.Vafa, D-Strings on D-Manifolds, HUTP-95/A035, IASSNS-HEP-95-77, hep-th/9510225

[5] Ooguri and C. Vafa., Two-Dimensional Black Hole and Singularities of CY Manifolds, hep-th/9511164

[6] A. Sen, A Note on Marginally Stable Bound States in Type II String Theory, MRI/PHY/23-95, hep-th/95102229

[7] A. Sen, U-duality and Intersecting D-branes, MRI/PHY/23-95, hep-th/9511026

[8] C. Vafa, Gas of D-Branes and Hagedorn Density of BPS States, HUTP-95/A042, hep-th/9511088

[9] E. Witten, Nucl. Phys. B443 (1995) 85

[10] A. Strominger, Nucl. Phys. B451 (1995) 96

[11] M. Bershadsky, A. Johansen, V. Sadov and C. Vafa, Nucl. Phys. B448 (1995) 166

[12] P. Horava, Phys. Lett. B231 (1989) 251

[13] J. Dai, R. G. Leigh and J. Polchinski, Mod. Phys. lett, A4 (1989) 2073

[14] J. Polchinski, Phys. Rev, D50 (1994) 6041

[15] J. Hughes, J. Liu and J. Polchinski, Phys. Lett. B180 (1986) 370

[16] K. Becker, M. Becker and A. Strominger, Fivebranes, membranes and Non-Perturbative StringTheory, Preprint NSF-ITP-95-62, hep-th/9507158

[17] D. D. Joyce, Compact Riemannian 7-manifolds with Holonomy $G_{2}$ : I, II, Oxford preprint; to appear in J. Diff. Geo. ;

Compact Riemannian 8-manifolds with Holonomy Spin(7), Oxford preprint; to appear in Inv. Math.

[18] S. Shatashvili and C. Vafa, Selec. Math. 1 (1995) 347

[19] R. Harvey and H. B. Lawson, Acta. Math. 148 (1992) 47

[20] R. Donagi, L. Ein and R. Lazarsfeld, A non-linear deformation of the Hitchin Dynamical System, preprint 1995

[21] H. Ooguri and C. Vafa, All Loop $N=2$ String Amplitudes, hep-th/9505183

[22] R. Lazarsfeld, J. Diff. Geom. 23 (1986) 299-307

[23] C. Vafa and E. Witten, Nucl. Phys. B431 (1994) 3

[24] C. Vafa and E. Witten, Nucl. Phys. B447 (1995) 261-270

[25] B. R. Greene, A. Shapere, C. Vafa and S.-T. Yau, Nucl. Phys. B337 (1990) 1

[26] R. C. McLean, Deformations and Moduli of Calibrated Submanifolds, Ph.D thesis, Duke University, 1990

[27] D. Joyce, private communication. 